\title{
ABSTRACTS OF CURRENT LITERATURE
}

\section{RESUSCITATION}

Outcome of Avalanche Victims with Out-of-Hospital Cardiac Arrest

Although prior studies have focused on avalanche victims with long durations of burial ( $>35$ minutes), few studies have targeted avalanche victims with short duration of burial $(<35$ minutes). In the absence of severe trauma, asphyxia is thought to be one of the main causes of cardiac arrest after avalanche burial. As the use of avalanche transceivers has increased, bystanders are more likely to locate victims quickly and begin resuscitation. The purpose of this study was to evaluate prehospital and in-hospital resuscitation efforts and ultimate outcomes for avalanche victims with a short duration of burial suffering out-of-hospital cardiac arrest.

Investigators performed a retrospective observational study from 2008 to 2013 from an avalanche accident registry in the Austrian Tyrolean Alps. Data were collected for prehospital care, in-hospital care, patient mortality, and neurological outcomes. A total of 55 victims were included in this study: 32 victims $(58 \%)$ were declared dead at the scene with no resuscitative efforts, all with either signs of severe trauma or prolonged burial time. Cardiopulmonary resuscitation (CPR) was performed on 23 victims (42\%), with only 2 victims experiencing a witnessed cardiac arrest. Nine of the victims receiving CPR had a short duration of burial ( $<35$ minutes). All of these 9 patients achieved return of spontaneous circulation (ROSC), 4 victims after bystander CPR and 5 victims after advanced emergency medicine care at the hospital. Five of the 9 victims survived to hospital discharge, but only 2 with full neurological recovery.

Although there was an overall $91 \%$ mortality for all patients included in the study with out-of-hospital arrest after an avalanche, the prognosis is slightly improved for victims with a short duration of burial and ROSC. Two of the 9 patients (22\%) who were buried for less than 35 minutes and achieved ROSC had a full neurological recovery. This study highlights the poor prognosis of out-of-hospital cardiac arrest for avalanche victims, but also identifies potential education for bystander rescuers. In the absence of severe trauma, firstresponders should focus on decreasing burial duration, providing adequate CPR, avoiding hypothermia, and expediting transfer to definitive care for avalanche victims.

(Resuscitation. 2015;89:114-118) L Moroder, B Mair, H Brugger, et al.

Prepared by Matthew Stewart, MD, FAWM, University of Utah Emergency Medicine Global Health Fellow, Salt Lake City, UT, USA.

\section{INDIAN PEDIATRICS}

Predictors of Myocardial Dysfunction in Children with Indian Red Scorpion (Mesobuthus tamulus) Sting Envenomation

Mesobuthus tamulus, the Indian red scorpion, is found in India, Pakistan, and Nepal, and its envenomation primarily targets the cardiovascular and pulmonary systems, resulting in myocardial dysfunction and pulmonary edema in severe cases. Prior studies have shown a decrease in pediatric mortality after scorpion envenomation with the administration of scorpion antivenom and prazosin; however, many victims still experience myocardial dysfunction. The goal of the current study was to identify risk factors for myocardial dysfunction in pediatric patients after Indian red scorpion envenomation to reduce overall morbidity.

The investigators conducted a prospective observational study at a tertiary care hospital in Southern India from 2012 to 2014. Inclusion criteria were patients younger than 13 years presenting with features consistent with scorpion sting envenomation. Myocardial dysfunction was defined as congestive cardiac failure, hemodynamic compromise, left ventricular dysfunction, elevated cardiac enzymes, and abnormal electrocardiogram. The study enrolled 85 children with an average age of 5.4 years. Overall, $28 \%$ of the patients experienced myocardial dysfunction, with significant predictive risk factors of hypotension at admission and increased time delay between envenomation and definitive treatment. Patients receiving antivenom and prazosin combination therapy had a decreased incidence of myocardial dysfunction when compared with patients receiving only prazosin.

The study emphasizes the importance of prompt referral and early and optimal treatment with scorpion antivenom and prazosin for pediatric patients presenting with high-grade Indian red scorpion envenomation to prevent myocardial dysfunction. Public health interventions include educating the public, referring facilities, and receiving facilities for prompt treatment after scorpion envenomation.

(Indian J Pediatr. 2015;52:297-301) A Kumar, S Krishnamurthy, R Srinivasaraghavan, et al.

Prepared by Matthew Stewart, MD, FAWM, University of Utah Emergency Medicine Global Health Fellow, Salt Lake City, UT, USA.

\section{EMERGING INFECTIOUS DISEASES}

\section{Animal-Associated Exposure to Rabies Virus among Tra-} velers, 1997-2012

Rabies is a continuing public health concern and causes approximately 60,000 deaths annually, primarily in Asia and Africa. Travelers are at continued risk of rabies exposure when visiting 